\title{
Spatial scale of correlated signals in 7T BOLD imaging
}

\author{
Andrew J Parker \\ University of Oxford \\ Dept Physiology Anatomy \& Genetics \\ Sherrington Bldg, Parks Road, Oxford, \\ OX1 3PT \\ Tel: +44 1865272504 \\ andrew.parker@dpag.ox.ac.uk
}

\author{
Holly Bridge \\ University of Oxford \\ FMRIB, Clinical Neuroscience \\ John Radcliffe Hospital \\ Oxford OX3 9DU \\ Tel: +44-1865 222716 \\ holly.bridge@ndcn.ox.ac.uk
}

\author{
Gaelle Coullon \\ University of Oxford \\ FMRIB, Clinical Neuroscience \\ John Radcliffe Hospital \\ Oxford OX3 9DU \\ Telephone number: +44 1865222493 \\ gaelle.coullon@ndcn.ox.ac.uk
}

\begin{abstract}
The spatial distribution of signals from magnetic resonance imaging (MRI) using measures of Blood Oxygen Level Dependent (BOLD) activations presents a fundamental limit on the ability of MRI to resolve the neural signals from the brain. Here we show that the multiple samples of low-level BOLD activity comprise a form of neural "imaging dust" with distinct spatial characteristics. We apply the distance-dependent measurement of variance to spatial maps of BOLD signals to deliver a new approach to estimating the empirical point-spread function (PSF) of MRI. We show that these new estimates are similar to earlier measures of the PSF of high field 7-T imaging, but deliver the advantage that they are specific to each individual tested in a single scanning session. We explore various potential applications of this approach.
\end{abstract}

\section{Categories and Subject Descriptors}

I.4.10 IMAGE PROCESSING AND COMPUTER VISION Image Representation; J.3 LIFE AND MEDICAL SCIENCES: Medical information systems

\section{General Terms}

Measurement, Performance, Experimentation.

\section{Keywords}

Magnetic Resonance Imaging, Visual Cortex, Point Spread Function, Variogram Analysis.

\section{INTRODUCTION}

Computational models of the visual cortex require the determination of several key parameters from experimental data. Chief among those parameters are the fundamental scales for the spatial and temporal organization of cortical tissue. These parameters are important in themselves for testing the plausibility of computational models of visual cortex. They are also important for linking together the outcome of diverse measurements of cortical function, which possess widely different sensitivities to spatial and temporal scale. Measurements such as patch-recording of single nerve cells tell us a great deal about the activity of single neurons at a cellular and sub-cellular scale, whilst imaging techniques pool together the signals from many neurons, often with the compensating advantage that organization on a larger spatial scale can be identified.

In this study, we apply a computational technique for extracting the spatial scale of correlated signals from high-field strength magnetic field imaging (MRI) in the living human brain, specifically the widely-used method of Blood Oxygen Level Dependent (BOLD) imaging of functional activity. BOLD imaging is an indirect measure of neural activity, which is generated when changes in neural activity lead to alterations in the oxygen content and flow of blood through the microvasculature of the cerebral cortex. Since the oxygenation state of the hemoglobin in the blood is accompanied by a change in magnetic state, measurements of BOLD within an MRI scanner can be achieved in living human cortex.

The temporal characteristics of the BOLD response have been well characterized, leading to the routine inclusion of a canonical hemodynamic response function in many software toolkits for analysis of BOLD MRI data. The spatial characteristics of BOLD imaging are less well understood, although it is widely accepted that a single voxel of MRI data must include the activity of many tens of thousands of neurons, both excitatory and inhibitory. Current MRI experiments typically report the averaged activity of all identifiable voxels within a single functional cortical area (V1, V2, V3, V3a, V5/MT). The advent of MRI scanning at higher field strengths, currently 7-Tesla in many MRI systems, is driving a renewed interest in the spatial resolution of BOLD imaging. Of particular interest is the claim that it may be possible to resolve spatial structure within single cortical areas, in the form of clusters or columns of neurons with similar functional responses to the content of the visual stimulus $[1 ; 6]$.

Measurement of the spatial resolving power of BOLD signaling within the visual cortex has typically required the use of specific stimuli to generate a spatially defined pulse of cortical activity, whose spread function can be directly estimated. In this paper, we present an alternative route to estimate the point-spread function (PSF) of BOLD imaging [4;5], which can be extracted from the intrinsic variability within a data set and does not require the setting-up of special conditions. Our approach applies some of the highly developed software tools for measurement of geospatial statistics to the measurement of BOLD responses from MRI data.

\section{THE MODELING FRAMEWORK}

The approach to modeling the spatial dependency of the BOLD response that we use here exploits the underlying variability of imaging data across a region of interest (ROI). Variability of a 

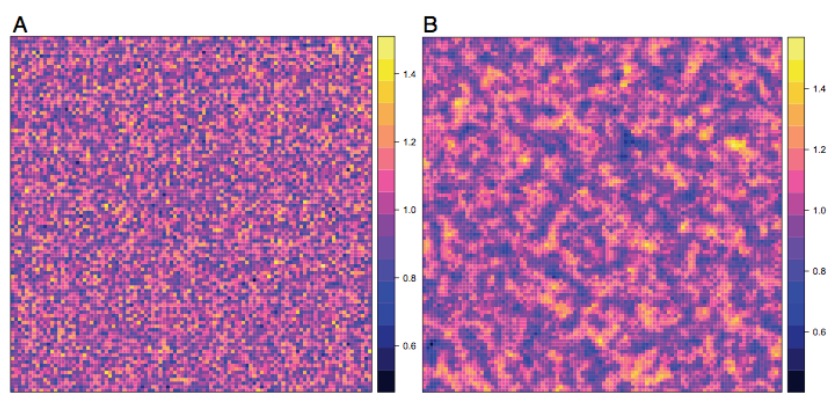

Figure 1: Fields of spatially uncorrelated (A) and spatially correlated (B) noise. The $x, y$ dimensions on these images are spatial distance across the cortex. The intensity (in arbitrary units) represents BOLD activity. The spatially correlated noise is a better model for the measured BOLD.

single sample point is characterized over repeated trials by the moments of the statistical distribution of responses, of which the second moment, the variance, is the most fundamental. Within the context of a field of spatial data, which have been sampled across many locations defined on a spatial co-ordinate system, it is possible to extend the idea of the variance of responses at a single sample point to the variance of the responses between two sample points $(x, y)$ and $(x+d x, y+d y)$. Fundamental to our approach is to consider how measured variance changes as a function of the spatial separation $(d x, d y)$ between sample points. Consider the two samples from noisy statistical distributions in Figure 1. In Figure 1A, the spatial noise sample is uniform white noise, such that the response at each and every sample point is independent from the response at other sample points. In Figure 1B, the noise is spatially structured such that responses at nearby sample points are more likely to be similar to each other than the responses at long-range distances across the spatial field. In the first case, there is no change in the variance between responses of pairs of sample points as a function of their separation $(d x, d y)$, whilst in the second case there is a strong spatial dependency of the variance between sample points.

\subsection{Variogram analysis}

The consequence of this spatial dependency is that the variance measured between nearby sample points is lower than the variance measured between more widely separated sample points. This spatial dependency of variance in many data sets describing natural phenomena has been characterized quantitatively, especially in the field of geospatial mapping. The variogram for a data set can be characterized as follows:

$\gamma(d x, d y)=1 / 2 \mathrm{E}\left[\langle Z(x+d x, y+d y)-Z(x, y)\rangle^{2}\right]$,

Equation 1

where $Z(x, y)$ is the activation at point $(x, y)$ and $\mathrm{E}[\mathrm{]}$ is the statistical expectation operator. Note that $\gamma$, the variogram, also sometimes called semivariance because of the factor of $1 / 2$, depends only on the separation of points $(d x, d y)$ and not the actual location of the points $(x, y)$.

The typical form for the variogram shows a rise in variance as the spatial separation between sample points increases. In data fields such as Figure 1B, the values of data from nearby sample points are correlated. This correlation declines with spatial separation, such that the measured variance rises. Characterization of the variogram therefore tells us something about the size and spatial scale of local spatial correlations in the data set.
In BOLD imaging data, these local correlations arise owing to a variety of sources that induce them upon the measurements. These include structurally static effects, such as signal drop-out at locations with high curvature in the cortical grey-matter. There are also functional effects, some arising from the blurring imposed by neurovascular coupling and others arising from local covariation in the underlying neural signals. The functional correlations may be expected to change in form, depending upon the visual stimulation applied during the data acquisition. Figure 2 shows the schematic form of a variogram as a function of spatial distance between the sample points next to a schematic form of the decrease in correlation with spatial distance. The latter function is of course equivalent to an autocorrelation function. The variogram can be applied to data that is sampled at irregular intervals because the data are treated as samples from a statistical point process.

\subsection{Spatial Autocorrelation of BOLD}

We apply this approach to high spatial resolution MRI data. The experimental details of the data acquisition are presented in the next section. Here we focus on the steps to estimate the variogram from a so-called "flat map" representation, in which the cortical activation of each point on a 2-D map of the unfolded occipital cortex is the starting point. Modeling of noise patterns was carried out using the R statistical software system [R version 3.1.2 (2014-10-31)] with several extension packages (notably 'fields' version 8.2-1 and 'gstat' version 1.0-21 available from the CRAN source http://cran.r-project.org) [3]. Analysis of the empirical distribution of variance in the MRI images was performed using the vgram function from the fields package. Figure 3A shows such a cortical flat-map, in which the activation is shown as a heat-map (from dark-red, through red and yellow, to white). The characteristic spatial structure of the activation is evident. This spatial structure is captured by the variogram (Figure 3B and 3C).
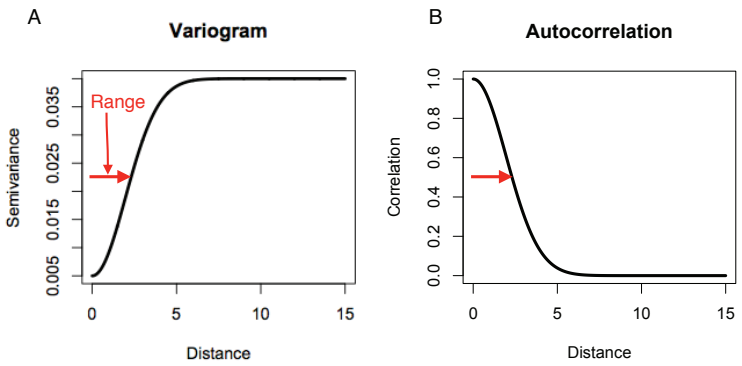

Figure 2: Relationship between variogram (A) and autocorrelation (B). As variance rises with distance (A), the correlation between sample points falls, according to the shape of the autocorrelogram (B). The range (red arrow) quantifies the spatial extent of correlations. For a stochastic noise process, the variogram directly predicts the autocorrelogram and vice versa.

\section{MRI OF VISUAL CORTEX}

\subsection{Image Acquisition}

Imaging data were collected on a 7T Philips Achieva MR system (Philips Healthcare, Best, Netherlands) using a volume transmit coil and a 32-channel receive coil (Nova Medical, Wilmington, MA), installed at Sir Peter Mansfield Magnetic Resonance Centre, University of Nottingham, UK. To minimize 
head motion, we stabilized participants with a customized MR compatible vacuum pillow (B.u.W. Schmidt) and foam padding.

Functional MRI data were obtained using $\mathrm{T}_{2} *$-weighted, gradient echo, three-dimensional echo-planar imaging with the following parameters: $\mathrm{TR}=88 \mathrm{~ms}, \mathrm{TE}=28 \mathrm{~ms}, \mathrm{FA}=22^{\circ}$, EPI factor 45. We used parallel imaging (SENSE reduction factor of 2.35 in the anterior-posterior (AP) direction and 2 in the foothead $(\mathrm{FH})$ direction [2]). Each complete volume acquisition (dynamic scan time) took $4 \mathrm{~s}$. The spatial resolution of the acquired data was $0.7 \mathrm{~mm}$ isotropic and the field of view (FOV) of the imaging volume was $74 \mathrm{~mm}, 36 \mathrm{~mm}$, and $154 \mathrm{~mm}$ in the anterior-posterior, foot-head and right-left directions respectively. The reduced FOV in the phase encoding direction (AP) required the use of outer-volume suppression to prevent signal fold-over. Standard forms of simulation [4] were performed to estimate the PSF directly from the imaging parameters (Matlab 8.0, Mathworks, Natick, MA), assuming a $\mathrm{T}_{2}{ }^{*}$ of $25 \mathrm{~ms}$ for gray matter, and resulted in a full-width-halfheight of less than 1.4 voxels, giving an effective resolution of approximately $1 \mathrm{~mm}$ in the phase encoding direction.

\subsection{Visual Stimulation}

The experimental MRI data were used to investigate activation to stereoscopic disparity in occipital cortex. Viewed monocularly, the stimuli consisted of patches of light and dark red and green random dots, to which binocular disparity could be applied. All stimuli were presented using a projector, viewed by the subject on a screen at their feet through red/green anaglyph prism glasses. The viewing distance was $240 \mathrm{~cm}$, and the stimuli subtended a visual angle of $10.75^{\circ}$. The stimulus was divided into upper and lower visual fields and equal and opposite disparity was added to the planes. At the start of a cycle of the experiment, the upper plane contained a positive disparity such that it was behind of the fixation plane. Every $2 \mathrm{~s}$ the plane moved away from the observer by a disparity step of $0.033^{\circ}$, passing through the fixation plane to a negative disparity of $0.215^{\circ}$. The lower plane did the opposite, starting at a near disparity plane of $-0.215^{\circ}$ and moved to $+0.215^{\circ}$ in counterphase with the upper plane. At the end of the $28 \mathrm{~s}$ cycle, the upper and lower planes jumped back to the starting disparity values and the cycle began again. Each scan consisted of 6 cycles, giving a total scan length of $168 \mathrm{~s}$. There were 8 scans in each session.

\section{QUANTIFYING THE SPATIAL CORRELATION OF BOLD SIGNALS}

The MRI experiments provide a cortical activation map, in which the BOLD signal varies across the spatial distance of the cortex. Figure 3A shows this for one subject S1 across the unfolded occipital cortex. Data were analysed using a Fourier analysis analogous to that used in retinotopic mapping. The response amplitude and phase of the best-fitting harmonic for each voxel were calculated. The coherence of response to the fundamental harmonic of the stimulus waveform was extracted. A high temporal coherence indicates that the BOLD signal is being strongly driven by the visual stimulus. We set a coherence threshold of 0.3, as adopted in other periodic analyses of BOLD activations. For main experimental study, the phase of high coherence responses is of primary interest, reflecting the disparity to which the voxel responded preferentially. However, for the purposes of the spatial correlation analysis here, we temporarily set aside the responses of voxels with amplitudes greater than the threshold of 0.3 . These voxels prove to have a different spatial correlation structure, potentially indicative of cortical regions with spatially clustered responses to the binocular disparity of the visual stimulus.

The focus here is on the large number of voxels whose activity is weakly linked to the visual stimulus, but which nonetheless have significant spatial correlation structure. Moreover, these voxels may have strong BOLD signals, although these signals are not strongly related to the changing disparity within the stimulus. Although the stimulus-related signal is weaker, there are a large number of voxels available for pairwise comparison to calculate the spatial distribution of variance and plot the result as a variogram. This smooths out a great deal of the potential variability in the analysis and provides a clean measure of the change in correlation with spatial distance. This distance dependency of correlation can be interpreted as a measure of the local PSF of the imaging measurements (see Figure 2). It is an empirical, rather than a theoretical, PSF. The distance dependence of the PSF also depends upon the quality of the cortical flattening that is achievable, since this process introduces some local distortions of spatial co-ordinates with respect to the MRI native 3-D space. Usefully, however, the empirical measure requires no special stimulus arrangements to extract the PSF from the data. The empirical measure also includes all local physiological sources of variability, whereas the theoretical PSF calculated from the imaging parameters provides a measure that is only strictly applicable to test objects, such as water-filled cavities [4].

The summary in Figure 3D shows the range, as specified by fitting the Gaussian curve. In effect, these values are the halfwidth at half-height of the PSF and may be compared with earlier estimates [5], which measured $1.17 \mathrm{~mm}$. However, Shmuel et al [5] selected a favorable location for direct measurement of the PSF and took specific precautions to avoid the signal from large blood vessels. No such steps were taken here. As noted earlier, direct estimates of the PSF from the imaging parameters used for these measurements give halfwidths slightly less than one $0.7 \mathrm{~mm}$ voxel. The empirical measures we have extracted here from the variogram analysis represent the practical limits on resolution that can be achieved with each data-set, taken individually for each subject and each experimental condition, including of course the number of repeated measures in a single session of scanning.

\section{DISCUSSION}

The theoretical PSF of BOLD imaging can be calculated from the imaging parameters for a particular scan sequence, whilst experimental measurements can deliver estimates of the PSF for specific imaging conditions $[4 ; 5]$. Although the approach here is indirect and folds in together multiple sources of local correlations in the spatial structure of the BOLD signal, the advantage is that the measures are directly relevant to the scanning of a particular individual within a specific scan session.

As mentioned earlier, the statistical power of this approach to estimation of the PSF depends upon the accumulation of multiple sources of weak evidence that combine to make a single smooth estimate. Our analysis here has purposefully focused on the low-level signals that are available. When we analyze the BOLD spatial maps, switching focus to high strength signals, then spatial structure on a larger scale is revealed. The fitted Gaussian curves widen to some $60 \%$ larger on average. Some of this structure may be due to static features 


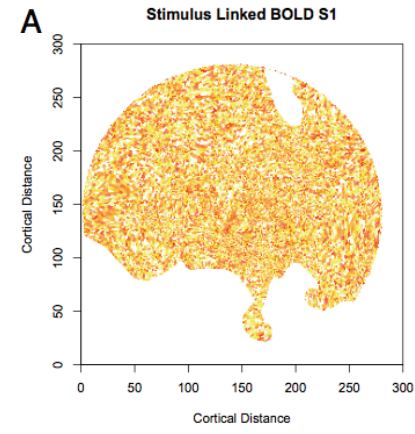

B
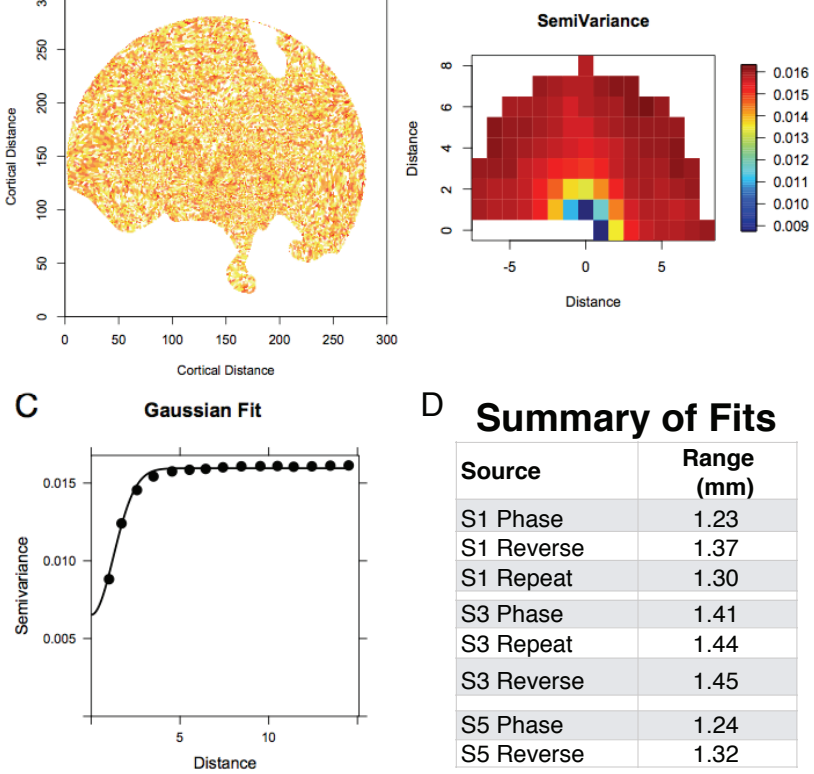

analysis to 3-D statistics, thereby allowing an analysis of MRI signals in their native space. This will allow the elimination of any spatial distortions induced by the flattening algorithms. Second, another development that we are exploring is obtaining spatial correlation measures that are site-specific to particular regions of interest (ROI) in the brain. In our case, this will mean the identification of ROIs within single visual cortical areas, such as V1, V2, V3, V3A etc., such that location-specific variograms can be calculated. Third, because the measures of resolution are derived from spatial fluctuations in the BOLD response, the same analytical approach can in principle be applied to so-called resting-state data, in which no specific sensory input or task is delivered.

This work was supported by grants from the MRC (G0802171, MR/K014382/1), Wellcome Trust (101092/Z/13/Z), a fellowship to HB from the Royal Society and a studentship to GC from St John's College Oxford.

\section{REFERENCES}

Figure 3: shows the steps in processing the variogram from MRI data. Fig3A: a flattened map of BOLD activity correlated to disparity waveform of stimulus is obtained for subject S1. Fig3B: semivariance is calculated in different directions relative to sample point according to Equation 1; the data are isotropic. Fig3C: radially symmetric variogram with Gaussian curve fitted. Fig3D: summary table of range parameter (see Figure 2) for different subjects S1, S3, S5. For "Reverse", the stimulus waveform followed the opposite sequence of disparity presentation and "Repeat" is a simple replica of the "Phase" condition. All distances are in units of $0.7 \mathrm{~mm}$ average voxel size, except summary table. The computation of the empirical variogram can be performed in different directions relative to the reference sample point. For some datasets, there will be an anisotropy in that spatial correlation will fall off more quickly in one direction rather than another. For these BOLD data at low amplitude signals, there is no evidence of anisotropy (Figure 3B), so radial symmetry around the sample point is used to calculate the empirical variogram in Figure 3C. The rise in variance with spatial distance is fitted with a Gaussian curve to quantify the range over which spatial correlations fall off.

such as larger blood vessels, but other spatial structure appears to indicate the presence of clustered groups of neurons representing similar features in the visual input. Specifying the base level of spatial correlation, as achieved in this paper, is essential for the correct interpretation of larger scale structure in the spatial map of BOLD signals.

The approach to measurement of MRI signals offered here opens up a number of interesting possibilities. First, methods based on geospatial statistics are not limited to data sampled across a 2-D surface. It is possible to extend the variogram
GONCALVES, N.R., BAN, H., SÁNCHEZPANCHUELO, R.M., FRANCIS, S.T., SCHLUPPECK, D., and WELCHMAN, A.E., 2015. 7 Tesla fMRI Reveals Systematic Functional Organization for Binocular Disparity in Dorsal Visual Cortex. The Journal of Neuroscience 35, 7 (February 18, 2015), 3056-3072. DOI= http://dx.doi.org/10.1523/jneurosci.3047-14.2015.

[2] PRUESSMANN, K.P., WEIGER, M., SCHEIDEGGER, M.B., and BOESIGER, P., 1999. SENSE: Sensitivity encoding for fast MRI. Magnetic Resonance in Medicine 42, 5, 952-962. DOI= http://dx.doi.org/10.1002/(SICI)15222594(199911)42:5\%3C952::AIDMRM16\%3E3.0.CO;2-S.

[3] R-DEVELOPMENT-CORE-TEAM, 2005. $R: A$ language and environment for statistical computing. $\mathrm{R}$ Foundation for Statistical Computing, Vienna, Austria. [4] ROBSON, M.D., GORE, J.C., and CONSTABLE, R.T., 1997. Measurement of the point spread function in MRI using constant time imaging. Magn Reson Med 38, 5 (Nov), 733-740.

[5] SHMUEL, A., YACOUB, E., CHAIMOW, D., LOGOTHETIS, N.K., and UGURBIL, K., 2007. in human gray matter at 7 Tesla. Neuroimage 35, 2, 539-552.

$\mathrm{DOI}=$ http://dx.doi.org/10.1016/j.neuroimage.2006.12.030.

[6] YACOUB, E., HAREL, N., and UĞURBIL, K., 2008. High-field fMRI unveils orientation columns in humans. Proceedings of the National Academy of Sciences 105, 30 (July 29, 2008), 10607-10612. DOI= http://dx.doi.org/10.1073/pnas.0804110105. Spatio-temporal point-spread function of fMRI signal 